\title{
Determinantes da não utilização de frameworks de gestão e/ou governança de
} TI

Humberto Caetano Cardoso da Silva

Doutorando em Administração pela Universidade Federal de Pernambuco

Professor da Uninassau Recife

humberto.ccs@gmail.com

Marcus Augusto Vasconcelos Araújo

Doutor em Administração pela Universidade Federal de Pernambuco.

Professor Adjunto da Universidade de Pernambuco e do Mestrado Profissional em Gestão Empresarial da Devry/FBV (MPGE/FBV).

Marcusaugusto77@hotmail.com

Jairo Simião Dornelas

Doutor em administração pela Universidade Federal do Rio Grande do Sul.

Professor do departamento de Administração e do Programa de Pós-Graduação em Administração da Universidade Federal de Pernambuco jairo@upe.br

\section{Editor Científico: José Edson Lara}

Organização Comitê Científico

Double Blind Review pelo SEER/OJS

Recebido em 14.06.2017

Aprovado em 29.11.2017

\section{(c) (1) (9)}

Este trabalho foi licenciado com uma Licença Creative Commons - Atribuição - Não Comercial 3.0 Brasil 


\title{
Resumo
}

A dependência da Tecnologia da Informação ( $\mathrm{Tl}$ ) faz com que este departamento se torne estratégico. Frameworks internacionalmente aceitos, de gestão e/ou governança de TI, são indicativos para o estabelecimento da boa governança de TI. Entretanto, a real utilização destas ferramentas é, ainda, pouco difundida, fazendo com que as empresas percam competitividade. $O$ presente estudo avaliou a utilização de frameworks de gestão e/ou governança de TI, internacionalmente reconhecidos, em empresas do nordeste Brasileiro, identificando os motivos pelos quais estes frameworks não são utilizados. A pesquisa foi realizada em duas etapas, a primeira utilizando um questionário direcionado e a segunda a partir de entrevistas semiestruturadas. Os resultados apontam que o distanciamento entre a direção e a $\mathrm{TI}$, a cultura organizacional, a baixa qualificação dos profissionais de TI e a falta de qualificação da direção da empresa em relação aos frameworks, são fatores da não utilização.

Palavras-chave: Tecnologia da Informação; Governança de TI; Gestão de TI; ITIL; COBIT.

\section{Determinants of non-use of IT governance and/or governance frameworks}

\begin{abstract}
The dependence of Information Technology (IT) makes this department strategic. Internationally accepted frameworks for IT management and / or governance are indicative of establishing good IT governance. However, the real use of these tools is still scarce, causing companies to lose competitiveness. The present study evaluated the use of internationally recognized management and / or IT governance frameworks in Brazilian Northeastern companies, identifying the reasons why these frameworks are not used. The research was carried out in two stages, the first using a directioned questionnaire and the second from semi-structured interviews. The results point out that the distance between management and IT, the organizational culture, the low qualification of IT professionals and the lack of qualification of the company managers in relation to the frameworks, are factors of non-use.
\end{abstract}

Keywords: Information Technology; IT Governance; IT Management; ITIL; COBIT.

\section{Determinantes de la no utilización de los frameworks de gestión y/o gobernanza de TI}

Resumen 
La dependencia de la Tecnología de la Información (TI) hace que el departamento se vuelva estratégico. Los estándares internacionales aceptados, de gestión y / o gobernanza de $\mathrm{TI}$, son indicativos para el establecimiento de la buena gobernanza de TI. Sin embargo, la utilización real de estas herramientas es, todavía, poco difundida, haciendo que las empresas pierdan competitividad. El presente estudio evaluó la utilización de frameworks de gestión y / o gobernanza de TI, internacionalmente reconocidos, en empresas del nordeste brasileño, identificando los motivos por los cuales estos frameworks no son utilizados. La investigación se realizó en dos etapas, la primera utilizando un cuestionario dirigido y la segunda a partir de entrevistas semiestructuradas. Los resultados apuntan que el distanciamiento entre la dirección y la TI, la cultura organizacional, la baja cualificación de los profesionales de TI y la falta de calificación de la dirección de la empresa en relación a los frameworks, son factores de no utilización.

Palabras clave: Tecnología de la Información; Gobernanza de TI; Gestión de TI; ITIL; COBIT.

\section{Introdução}

A grande competitividade nos mercados vem forçando as empresas a serem cada vez mais eficientes, e a utilização da Tecnologia da Informação (TI) é uma das ferramentas que pode trazer um diferencial e possibilitar vantagem competitiva. Os altos investimentos realizados em $\mathrm{TI}$, a grande dependência que as empresas têm em relação à $\mathrm{TI}$, algumas delas de cunho legal, a capacidade de melhoria na comunicação da empresa com o cliente e a possibilidade da criação de novos produtos e serviços, faz com que o setor de TI das empresas tenha um papel estratégico (Lunardi, Dolci, Maçada, \& Becker, 2014; Bianchi, 2015).

A governança de uma organização representa a capacidade que os seus dirigentes têm de assegurarem, de forma consistente e previsível, que a organização consiga atingir seus objetivos sociais e suas obrigações legais. Uma governança eficaz de TI faz com que a gestão da empresa e a utilização de elementos de TI estejam em harmonia e que os objetivos de negócio sejam alcançados (Weill \& Ross, 2006).

Ainda que seja um setor importante para o bom desempenho empresarial, a gestão e a governança de TI têm sido deixadas ao acaso. Kaur e Sengupta (2011) argumentam que, em média, $70 \%$ dos projetos envolvendo $\mathrm{TI}$ falham em atingir os seus objetivos e que nos Estados Unidos cerca de 30 bilhões de dólares são perdidos devido a essa elevada taxa de falhas na entrega dos projetos. Complementarmente, 
um estudo do Standish Group (2014), com mais de 8380 projetos em mais de 800 empresas, revelou que apenas $16 \%$ dos projetos em TI foram implementados com sucesso, $31 \%$ deles foram deixados incompletos, e $53 \%$ deles tiveram custos e prazos maiores que os planejados (Zaitar \& Ouzarf, 2012).

A utilização de frameworks como COBIT, ITIL, PMI, etc., é um dos mecanismos que pode desencadear um comportamento organizacional consistente, alinhando os investimentos em $\mathrm{Tl}$ com as reais necessidades da empresa, proporcionando melhores resultados e maior visibilidade da TI como um todo (Weill \& Ross, 2005).

Apesar da necessidade de uma melhor gestão e uma governança eficaz, a adoção de frameworks de sucesso reconhecido internacionalmente é pequena. Em um levantamento do IDC com 225 organizações, 43,6\% delas utilizavam "padrões próprios", 27,1\% utilizavam ITIL e 12,9\% utilizavam COBIT (Broussard \& Tero, 2007). Outro estudo feito por Tarantino (2008) revelou que 33\% das empresas utilizam "padrões próprios", 13\% ITIL e 9\% COBIT. Apesar dos números discrepantes, a fato da baixa aderência a modelos internacionalmente aceitos é presente nos dois estudos.

Complementarmente, a utilização de "padrões próprios", aqui definidos como uma forma própria desenvolvida localmente na empresa para governar e gerir $\mathrm{TI}$, tem pontos negativos como a necessidade de treinamento dos novos membros da equipe, pois os padrões desenvolvidos na empresa não são utilizados em outros locais, a dificuldade de aplicar esses padrões em outras empresas e mesmo dentro da empresa caso esta precise mudar, e a utilização de procedimentos ad hoc, mais demorados e imprecisos para a criação dos processos a serem utilizados (OGC, 2007).

Assim, considerando a necessidade de gestão e governança da $\mathrm{TI}$, o presente estudo tem como objetivo avaliar a utilização de frameworks de gestão e/ou governança de $\mathrm{Tl}$ em empresas e identificar os motivos pelos quais frameworks internacionalmente reconhecidos não são utilizados na gestão e/ou governança de TI em detrimento a procedimentos ditos como "padrões próprios".

Este trabalho está estruturado em quatro sessões. Inicialmente será discutida a fundamentação teórica, na qual discutiremos a Governança Empresarial e a Governança de TI e os Frameworks de TI. A segunda parte apresenta os procedimentos metodológicos do estudo. $\mathrm{Na}$ terceira parte serão discutidos os 
resultados encontrados na pesquisa. E, finalmente, na quarta parte serão apresentadas as conclusões finais do estudo.

\section{Fundamentação Teórica}

\subsection{Governança e gestão de TI}

A dependência de $\mathrm{Tl}$ e os riscos associados aos sistemas computacionais são fatores que tornam críticos a necessidade de governança e gestão da área de TI. A informação é um ativo importante na organização, e sua gestão protege não somente a tecnologia, mas os negócios a empresa em si. A TI está focada na criação de valor de negócios, mas a preservação deste valor é papel da governança, que implementa os controles para a identificação de possíveis causas de problemas futuros, eliminando, transferindo ou mitigando acontecimentos indesejáveis (Lunardi, Becker, \& Maçada, 2010).

A governança corporativa tem um papel decisivo no desenvolvimento da governança de $\mathrm{TI}$, sendo este um subconjunto da governança corporativa (ITGI, 2003). As corporações modernas são caracterizadas pela separação entre o controle e a gestão, fazendo com que surjam novos papéis como o do acionista. Neste contexto a governança corporativa possibilita a transparência, equidade, prestação de contas e a responsabilidade corporativa necessárias nesse ambiente de vários stakeholders ${ }^{l}$ (Lunardi, Becker, \& Maçada, 2014).

A governança de $\mathrm{Tl}$ é de responsabilidade da direção da empresa e não apenas da organização de TI. A governança de TI consiste na direção, estrutura organizacional e processos que garantam que TI sustente e amplie objetivos e estratégias organizacionais (ITGl, 2003). Não é uma preocupação com a localização ou distribuição de recursos, mas a utilização de padrões gerenciais de responsabilidade objetiva e controle, de forma que os recursos aplicados possam dar o retorno esperado (Weiss \& Bernardes, 2014).

A implantação de novas práticas de governança de TI requer mudanças na forma de operar da organização, mudanças no modus operandi. A gestão dos aspectos relacionados a riscos é fundamental para que os resultados sejam positivos.

1 - Stakeholder: Pessoa ou grupo que fez um investimento ou tem ações ou interesse em uma empresa, negócio ou indústria. 
Isto requer uma avaliação das características organizacionais e culturais da empresa, de forma que estes não se tornem empecilhos na implantação do novo modelo de trabalho. Não basta apenas documentar os processos, é necessária uma nova visão do fluxo operacional e a implementação de práticas funcionais (Weiss \& Bernardes, 2014).

O objetivo da governança de $\mathrm{Tl}$ é o alinhamento, a integração e a relação entre os vários setores da organização, seus stakeholders e TI. É necessário que exista uma compreensão do que é realizado por TI, quais seus objetivos e como identificar quando as metas forem alcançadas e o que esperar para as novas metas (Creamer \& Freund, 2010).

Para o ITGI (2007) cinco pilares são necessários para que se possa fundamentar um efetivo modelo de governança de TI. São eles:

Apêndice A - A compreensão clara do ambiente empresarial, interna e externamente, de forma que se conheça o que é exigido de TI; dessa forma a Tl e os serviços que ela presta estarão alinhados aos elementos do ambiente corporativo;

Apêndice B - Entrega de serviços de TI no prazo, dentro do orçamento e com as melhorias esperadas, de forma que a TI possa entregar de valor;

Apêndice C - Gerenciar o risco, abrangendo todos os processos de preservação de valor;

Apêndice D - Gerenciar os recursos, estabelecendo e implantando as capacidades de TI exigidas para atendimento das necessidades do negócio;

Apêndice E - Gerenciar o desempenho, de forma que se possa garantir que o departamento de TI esteja alinhado ao negócio.

O ITGI (2007) também argumenta que a governança de TI possibilita a institucionalização de boas práticas de forma a assegurar que a informação e a tecnologia relacionada ajudem a empresa a atingir seus objetivos. Garantir que os investimentos realizados em tecnologia estão adequados aos objetivos e necessidades da organização é o objetivo do gerenciamento de recursos de TI. Estes recursos incluem hardware, software, habilidades humanas e processos de gerenciamento, de forma que os investimentos em TI sejam traduzidos em desempenho da TI (Lunardi, Becker, \& Maçada, 2010). 
Manter e entregar os serviços de Tl de forma que estes estejam em sintonia com as necessidades da empresa é o objetivo do gerenciamento de serviços de $\mathrm{Tl}$. Além disso é necessário que os serviços de TI possibilitem maior competitividade à organização sendo mais confiáveis, disponíveis, seguros e comportem eventuais mudanças de direção e flutuações de mercado. Otimizando e controlando os custos de Tl é possível adicionar valor efetivo aos serviços que este oferece, fazendo com que TI se torne parte integrante do negócio (Weiss \& Bernardes, 2014).

\subsection{Frameworks de TI}

Vários são os frameworks para gestão ou governança de TI. Em gestão de infraestrutura de TI o framework mais utilizado é o ITIL V3 2011. Já na área de controladoria e auditoria o COBIT, "Control Objectives for Information and Related Technologies" é o framework mais utilizado. (Lyra, 2013; Bianchi, 2015).

Um framework é uma estrutura conceitual básica, ou uma estrutura de instruções, destinada ao auxílio na implantação, suporte, manutenção gerenciamento e controle de uma infraestrutura, no presente artigo, uma estrutura de TI (Ngulube et al., 2015). Alguns frameworks como o ITIL, o COBIT e o CMMI, alcançaram índices de uso internacionais e oferecem aos gestores de $\mathrm{TI}$ as melhores práticas para a gestão e o controle do parque tecnológico (Talea et al., 2015).

\subsubsection{ITIL}

A ITIL, acrônimo de "Information Technology Infrastructure Library", teve sua primeira versão lançada em 1989 pela Her Majesty's Stationery Office - HMSO no Reino Unido a pedido da Central Communication and Telecommunications AgencyCCTA (Agência Central de Comunicação e Telecomunicação). Sua última versão foi lançada em 2011 e o framework atualmente faz parte do Office of Government Commerce - OGC (Gabinete de Comércio do Governo) (Lyra, 2013).

A ITIL é uma abordagem para gerenciamento de TI, oferecendo orientações baseadas em melhores práticas, sendo um padrão em gestão de TI (Magalhães \& Pinheiro, 2007). A biblioteca é composta por cinco livros e 27 processos, que abordam todas as etapas do ciclo de vida dos serviços de TI, desde o planejamento estratégico 
até a entrega do serviço, observando a melhoria continuada dos serviços (Orr \& Britain, 2011; Lyra, 2013). Os cinco livros da biblioteca ITIL são:

- Estratégia do Serviço: É o ponto central do ciclo de vida do serviço. Este livro oferece orientações de como fazer com que o gerenciamento de serviços possa ser um ativo estratégico da empresa;

- Desenho do Serviço: Este livro fornece orientações de como projetar e desenvolver serviços e processos de gerenciamento dos mesmos. Analisando serviços novos e implementados, de forma que os objetivos estratégicos se tornem portfólios e ativos de serviços;

- $\quad$ Transição do Serviço: Aqui são abordados aspectos de direcionamentos para o desenvolvimento de melhorias e capacidades, de forma que serviços novos ou modificados possam entrar em operação com sucesso controlando os riscos;

- $\quad$ Operação do Serviço: É o ponto de entrega do serviço. Aqui são tratados procedimentos que permitam que os serviços possam ser entregues dentro dos níveis de serviço acordados (SLA - service-level agreement);

- Melhoria Continuada do Serviço: Tem como objetivo manter e criar valor por meio de melhorias ao serviço. Este livro atua em qualquer momento do ciclo de vida do serviço, podendo agir desde a Estratégia até a Operação.

A implementação do gerenciamento de serviços de TI se dá a partir de quatro necessidades: (1) quando é necessário que seja melhorada a eficiência organizacional e sejam reduzidos os gastos com TI; (2) quando a qualidade com foco na prestação de serviços precisa ser melhorada; (3) quando o alinhamento com os clientes e internamente entre o negócio e as funções da TI precisa ser alcançado, e (4) quando é necessário melhorar a qualidade do serviço e consequentemente a satisfação dos clientes (Iden \& Eikebrokk, 2013).

Cater-Steel, Tan e Toleman (2006) argumentam que são cinco os fatores de sucesso para implementação de gestão de TI, sendo: (1) comprometimento da alta administração; (2) possuir um profissional com capacitação em gestão de projetos; (3) habilidade da TI em se adaptar a mudanças; (4) competências da equipe de TI, e (5) qualificação e formação da equipe de TI. Pereira e Silva (2010) acrescentam que pontos como o tamanho da organização, orçamento disponível, formação e qualificação da equipe da organização e se esta é pública ou privada, 
devem ser levados em consideração no momento da implementação do framework.

Apesar de ser utilizado mundialmente para gestão de TI, a adoção das práticas ITIL em uma organização traz desafios e muitas vezes o processo não é bemsucedido. Implementar ITIL em uma organização é difícil, os riscos são altos e os projetos são longos e muitos desses falham (Pereira \& Silva, 2010).

\subsubsection{COBIT}

O COBIT (Control Objectives for Information and Related Technology) foi criado em 1994 pela ISACF (Information Systems Audit and Control Foundation) ligado à ISACA (Information Systems Audit and Control Association), que é uma associação internacional formada por profissionais que atuam nas áreas de auditoria de sistemas. O COBIT é um conjunto de objetivos de controle que estão alinhados com outros modelos e em conformidade com regulamentações e boas práticas da Governança de TI (ITGI, 2007).

O framework COBIT é um modelo internacionalmente aceito como um conjunto de melhores práticas para o exercício do controle sobre a informação, tecnologias e riscos a ela associados. Com ele é possível que seja realizada uma conexão entre as metas de Tl e os objetivos do negócio, gerenciar de forma transparente o desempenho de $\mathrm{TI}$, organizar Tl em um modelo de processos, identificar recursos importantes para que a TI possa desempenhar seu papel e definir metas que possam ser utilizadas no acompanhamento e gerenciamento das atividades da TI (Weiss \& Bernardes, 2014).

Segundo Laurindo (2008) os controles definidos pelo COBIT são políticas, procedimentos, práticas e estrutura organizacional a serem desenvolvidos de forma que os objetivos de negócio sejam atingidos de forma segura, e que eventos indesejáveis possam ser prevenidos, detectados e corrigidos. Nesta mesma linha, De Alcântara (2007) argumenta que o COBIT considera as informações corporativas e a tecnologia necessária para suportá-las são uma variável endógena da estratégia corporativa e que o modelo do COBIT tem como proposta cobrir todos os processos normalmente encontrados em $\mathrm{TI}$, detalhando tanto o que precisa ser feito quanto $\mathrm{o}$ que está, de fato, sendo realizado.

O COBIT, em sua versão 5, lançada em 2012, fez a integração de conceitos e princípios do COBIT 4.1 com outros frameworks como Val IT, Risk IT, BMIS e ITAF. 
Adicionalmente, o seu alinhamento com outros frameworks do mercado foi melhorado, especialmente com o ITIL. Segundo a sua nova versão, cinco princípios são fundamentais (ITGI, 2012):

- $\quad$ Princípio 1: Reunir as necessidades dos stakeholders ;

- $\quad$ Princípio 2: Cobrir a empresa fim-a-fim;

- $\quad$ Princípio 3: Aplicar um framework único e integrado;

- $\quad$ Princípio 4: Aplicar uma abordagem holística;

- $\quad$ Princípio 5: Separar a governança da gestão.

Na versão 5, o COBIT possui 5 domínios, dividindo o framework em governança e gestão, sendo o processo (1) Avaliar, Dirigir e Monitorar, específico da área de governança e os processos (2) Alinhar, Planejar e organizar, (3) Construir, Adquirir e Implementar, (4) Entregar, Serviços e Suporte, (5) Monitorar, Avaliar e Analisar, específicos da área de gestão. Ao todo o framework possui 37 processos (ITGI, 2012).

O COBIT tem sido utilizado como uma ferramenta para avaliar a utilização de metodologias e melhores práticas para o gerenciamento de $\mathrm{TI}$, além da sua aplicação em empresas que precisam atender aos requisitos da lei Sarbanes-Oxley (Lunardi et al., 2014). Entretanto, dentro desse universo de domínios e processos, Bianchi (2015) argumenta que existe uma dificuldade para os gestores compreenderem quais os processos devem ser implementados, e que além dos processos, que já são 37, cada um deles possui uma diversidade de objetivos de controle.

\section{Procedimentos Metodológicos}

Esta pesquisa, de caráter descritivo, teve como objetivo avaliar a utilização de frameworks de gestão e governança de $\mathrm{Tl}$ em empresas do nordeste brasileiro e identificar os motivos pelos quais frameworks internacionalmente reconhecidos não são utilizados na governança e gestão de $\mathrm{Tl}$ em detrimento a procedimentos ditos como "padrões próprios". Segundo Gil (2010), as pesquisas descritivas têm como objetivo a descrição das características de um fenômeno, no caso do presente estudo, da não utilização de frameworks internacionalmente reconhecidos.

A pesquisa foi realizada com empresas de setores variados da economia. A 
mesma foi dividida em duas partes. A primeira parte foi realizada através de um questionário direcionado, no qual os gestores de TI participantes respondiam se utilizavam ou não frameworks de governança ou gestão de TI. O questionário foi enviado para os participantes em janeiro e fevereiro de 2016, sendo estes profissionais de $\mathrm{TI}$ desde estagiários até diretores. Para cada empresa participante, 56 ao todo, apenas a pessoa responsável por TI foi convidada a responder o questionário. O objetivo dessa etapa foi identificar a utilização de frameworks de $\mathrm{TI}$ em empresas e se esta ferramenta, o framework de gestão, era devidamente difundida entre todos os participantes do setor, proporcionando uma aplicação completa dos mesmos.

O questionário foi criado pelos pesquisadores de forma que o participante respondesse o mesmo sem utilizar-se de termos conhecidos. Por exemplo, ao questionarmos um gestor de TI sobre a utilização de ITIL, este poderia responder que usa o framework apenas pela familiaridade com o termo.

As questões foram cadastradas em um sistema de formulários online que nos permitiu direcionar o respondente. Assim, caso o respondente não conheça o termo "framework de gestão ou governança de TI", que poderia ser ITIL, COBIT ou outro framework de padrão internacional ou local, ele seria direcionado para a pergunta "Você conhece os frameworks ITIL ou COBIT?". A partir da resposta dada, o participante da pesquisa poderia ser encaminhado para uma pergunta ou outra, que tinha como objetivo identificar o nível de conhecimento dos frameworks do estudo e se o participante realmente utiliza algum dos processos propostos pelos frameworks. O fluxograma com a lista de perguntas e os possíveis caminhos a serem seguidos está demonstrado na Figura 1. 


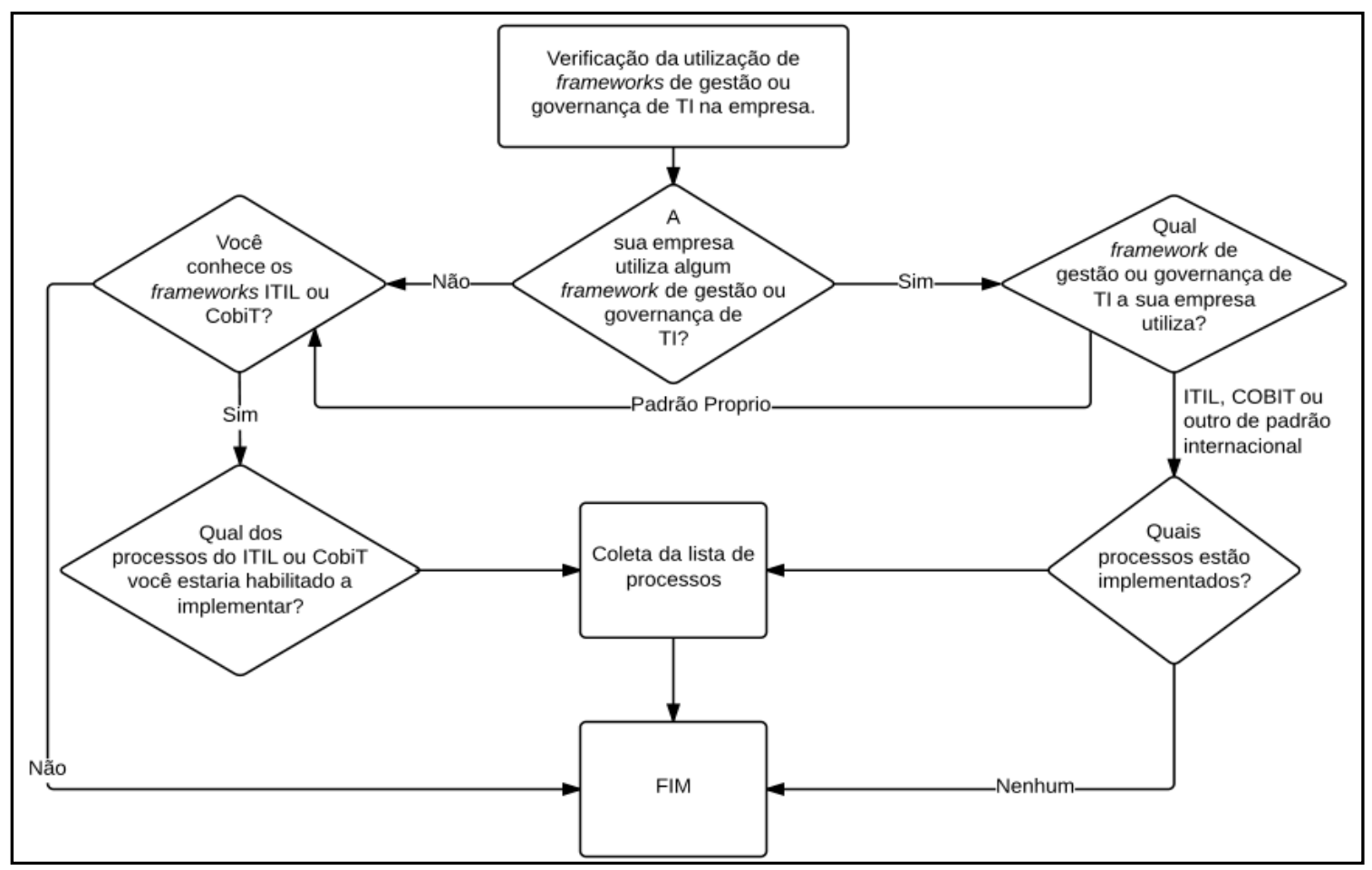

Figura 1

Fluxograma de pesquisa

Fonte: Elaborado pelos autores

A segunda parte do estudo foi realizada a partir de entrevistas semiestruturadas. Segundo Manzani (1990) e Gil (2010), entrevistas semiestruturadas focalizam um assunto com perguntas gerais, desta forma é dada liberdade ao entrevistado para falar livremente. Todas as entrevistas foram gravadas e, posteriormente, transcritas, de forma que os dados obtidos pudessem ser analisados.

As questões utilizadas na etapa qualitativa foram criadas pela equipe de pesquisa, a partir dos cinco fatores de sucesso propostos por Car-Steel, Tan e Toleman (2006), de forma que pudesse ser identificado qual o nível de utilização de frameworks de governança ou gestão de TI (Questão 1). Em seguida, identificar quais seriam as dificuldades encontradas na implantação desses frameworks (Questão 2). Conforme colocado pelo ITGT (2003), a governança de TI é de responsabilidade da direção da empresa, portanto é importante saber qual a posição da alta gestão em relação ao assunto (Questão 3). A própria equipe de TI poderia 
apresentar dificuldades no processo de implantação, sendo a falta de treinamento específico nas ferramentas uma delas (Questões 4 e 5). Finalmente, volta-se a questão da participação da alta administração, mas tomando como foco o treinamento do administrador em gerir e/ou governar TI (Questão 6).

As questões utilizadas nesta etapa foram:

1. A utilização de frameworks de TI é indicada como forma de aumentar a performance e melhorar a governança de TI. Neste cenário, qual o grau de utilização destes frameworks na sua empresa?

2. Qual a sua dificuldade em implementar e utilizar frameworks de governança ou gestão de TI?

3. Em algum momento a direção da empresa cobrou ou apoiou a utilização destes frameworks TI?

4. Com relação à equipe de $\mathrm{TI}$, qual a dificuldade na implantação de um framework de TI?

5. A equipe de TI já possuía alguma capacitação formal, ou teve o treinamento adequado, em algum framework de $\mathrm{TI}$ ?

6. O COBIT cobra a participação da alta direção da empresa na governança de $\mathrm{Tl}$, existe algum projeto para treinamento dos gestores nessa área?

Os sujeitos da segunda etapa do estudo foram gestores de Tecnologia da Informação. Este cargo foi escolhido, pois, são estes indivíduos que atuam diretamente com a direção e que aplicam as políticas e processos de gestão do setor de TI. O conjunto de sujeitos desta pesquisa foi definido pelo critério de acessibilidade, sendo realizado, entretanto, um esforço para que participantes de setores variados fizessem parte da pesquisa. Ao todo foram 5 entrevistados, sendo 1 empresa do setor de supermercados de âmbito regional (sujeito A), 1 do setor de varejo de artigos para o lar de âmbito regional (sujeito B), 1 de indústria têxtil de âmbito nacional (sujeito $\mathrm{C}$ ), 1 do setor de varejo de fármacos de âmbito regional (sujeito D), e 1 do setor de tele atendimento de âmbito nacional (sujeito E). As entrevistas foram realizadas nos meses de fevereiro e março de 2016.

As transcrições das entrevistas foram analisadas através da técnica de análise de discurso, com o objetivo de identificar os significados mais relevantes das 
entrevistas. Assim, a análise de discurso consiste em uma busca do conhecimento de uma construção do texto e fornece subsídios para lidar com os processos de constituição do fenômeno linguístico, e não meramente do seu produto, em análises de comunicações em geral (Cappelle, Melo, \& Gonçalves, 2011).

\section{Análise dos Resultados}

\subsection{Etapa quantitativa}

Após a realização da primeira etapa do estudo, obtivemos um total de 56 respondentes e verificamos que a pesquisa atingiu uma grande variedade de setores empresariais. O setor de serviços, com 29 respondentes, foi o setor que apresentou a maior quantidade de respondentes, seguido pelo setor de construção, com um total de 13 respondentes. A distribuição de respondentes por setor empresarial pode ser vista na Tabela 1.

\section{Tabela 1}

Lista de respondentes por setor empresarial

\begin{tabular}{l|c|c}
\hline \hline Setor & Quantidade & $\%$ \\
\hline Serviços & 29 & $52 \%$ \\
\hline Construção & 13 & $23 \%$ \\
\hline Indústria & 4 & $7 \%$ \\
\hline Comércio & 3 & $5 \%$ \\
\hline Atacado & 3 & $5 \%$ \\
\hline Público & 4 & $7 \%$ \\
\hline
\end{tabular}

Fonte: Dados da Pesquisa.

O número de funcionários de cada um variou desde apenas 4 , até empresas com mais de 10.000 funcionários. O número de funcionários é um fator importante na gestão de $\mathrm{TI}$, pois representa, a grosso modo, o número de usuários que o setor de TI precisa atender. As três maiores empresas do estudo, com 10.000, 7.000 e 5.000 funcionários, utilizam frameworks de gestão ou governança de TI. Em contrapartida, profissionais de empresas com 4.000, e duas com 2.000 funcionários relataram não os utilizar. A lista de empresas participantes do estudo que declararam (não) utilizar frameworks de gestão ou governança de TI pode ser visualizada na Tabela 2. 


\section{Tabela 2}

Utilização de Frameworks de gestão ou governança de TI

\begin{tabular}{l|c|c|c}
\multicolumn{2}{c|}{ Empresas participantes do estudo } & \multicolumn{2}{c|}{ Utilização de Frameworks de governança ou gestão } \\
\hline N. Funcionários & Quantidade & Sim & Não \\
\hline $4-50$ & 14 & 6 & 8 \\
\hline $51-100$ & 8 & 4 & 4 \\
\hline $101-200$ & 13 & 2 & 11 \\
\hline $201-500$ & 5 & 4 & 2 \\
\hline $501-1000$ & 4 & 2 & 4 \\
\hline $1001-2000$ & 5 & 1 & 2 \\
\hline $2001-10000$ & 7 & 5 & 32 \\
\hline Total & 56 & 24 & \\
\hline
\end{tabular}

Fonte: Dados da Pesquisa.

Foi possível identificar que $57,1 \%$ dos participantes da pesquisa disseram não utilizar nenhum framework de governança ou gestão de TI. Dos 42,9\% que declararam utilizar frameworks de governança ou gestão $29,2 \%$ disseram utilizar "padrões próprios". Ou seja, apenas 30,3\% dos participantes na pesquisa utilizam frameworks de gestão ou governança de TI internacionalmente reconhecidos. Entre aqueles que utilizam "padrões próprios" ou disseram não utilizar nenhum framework, 69\% conhecem ou já ouviram a respeito de ITIL, COBIT, ou outro padrão internacional de governança e gestão de TI.

Outra informação coletada na pesquisa foi que, mesmo relatando que possuem frameworks como ITIL ou COBIT implementados na empresa que trabalham, apenas $64 \%$ dos profissionais conseguiram descrever algum processo que está efetivamente implementado. Já entre os que utilizam "padrões próprios" e disseram conhecer ITIL ou COBIT, apenas $7 \%$ dos participantes da pesquisa afirmaram ter capacitação para implementar algum dos processos destes frameworks.

Este quadro indica o baixo nível de utilização destes frameworks internacionais em empresas no nordeste brasileiro, corroborando com pesquisas já realizadas anteriormente por Broussard e Tero (2007) e Tarantino (2008). Apesar de serem pesquisas realizadas há quase 10 anos, o quadro não mudou significativamente, continuando a gerar situações de baixa performance, ingerência e erros na condução da utilização da TI.

\subsection{Etapa Qualitativa}

Após a análise das transcrições das entrevistas, identificou-se que nas 
empresas nas quais os sujeitos $\boldsymbol{A}, \boldsymbol{B}, \boldsymbol{C}$ e $\boldsymbol{D}$, eram gestores a utilização de frameworks de gestão e/ou governança de TI é muito baixa, ou mesmo inexistente. Já no caso do sujeito $E$, a utilização destes frameworks é intensa, fazendo parte da cultura organizacional.

Os sujeitos $\boldsymbol{A}, \boldsymbol{B}, \boldsymbol{C}$ e $\boldsymbol{D}$ não contavam com o apoio da direção na implantação e manutenção de ferramentas de gestão e governança de $\mathrm{TI}$, demonstrando um claro distanciamento entre TI e os objetivos da empresa. Existe uma falsa impressão que Tl é uma área apenas técnica e que não pode contar com nenhum apoio gerencial da direção. $O$ sujeito $C$ relata que

Para a direção este assunto é indiferente. O mais importante é que as atividades computacionais da organização não parem e que a TI só tem que "...trocar o pneu do carro com ele em movimento...".

Já o sujeito $\boldsymbol{D}$ coloca que " $A$ direção não tem conhecimento desse tipo de tecnologia". Esta é uma afirmação interessante, pois os frameworks de gestão e governança não tem aspectos técnicos, mas administrativos, então o próprio gestor de Tl atribui a estes um aspecto técnico de maneira a justificar a ausência da direção no dia a dia do setor.

Este distanciamento entre TI e a direção da empresa também se apresenta na declaração do sujeito $B$ :

Existem conversas com a diretoria de uma apresentação de como é que funciona, como é que eles podem participar, como a diretoria pode participar e só depois disso é que eu vou saber o quanto é que o pessoal vai ficar disposto, a diretoria vai estar disposta a participar efetivamente. Eu acredito que não vá ser muito, não vão influenciar tanto, mas assim... eles têm sido bem positivos no que eu tenho levado para eles.

Declarações como estas estão presentes nas respostas dos entrevistados que não tem frameworks de TI implementados, ou que tem estes implementados apenas por iniciativa da equipe de TI.

Para o sujeito $E$, que tem processos de gestão e governança maduros e bem implementados, deixa claro a participação da diretoria na vida do departamento de $\mathrm{TI}$, cobrando, não aspectos técnicos, mas eficiência e clareza no andamento das 
demandas tratadas pelo pessoal de TI. Declara o sujeito $E$ que "somos cobrados por resultados, temos que bater nossa meta e somos cobrados por isso".

Um segundo ponto presente nas respostas dos entrevistados é a dificuldade em implementar os frameworks por conta da chamada "cultura organizacional". Três dos entrevistados, sujeitos $\boldsymbol{A}, \boldsymbol{B}$ e $\boldsymbol{C}$, citaram ser esse o motivo da dificuldade de implantação dos frameworks. $O$ sujeito $\boldsymbol{C}$ declarou que:

A Cultura Organizacional. Muitas empresas ainda caminham com passos lentos quando o assunto é investimentos em Processos de TI. Ainda existe um pouco de resistência para considerar que a área de TI possui suas atividades diárias importantes para o negócio e que estas atividades não devem ser negligenciadas. Acredito que ainda exista o pensamento que é só ligar o equipamento e pronto!

Já o sujeito $\boldsymbol{A}$ acrescenta que " $\boldsymbol{A}$ cultura das pessoas é de se envolver com o pessoal que dá mais a resposta, fazer mais o dia a dia acontecer e aceitar isso ai". Esta declaração do sujeito $\boldsymbol{A}$ está ligada a maneira desestruturada de trabalho que o setor de $\mathrm{Tl}$ de várias organizações vivencia diariamente. $O$ processo de estruturação destes setores é trabalhoso e requer dedicação e apoio. $O$ sujeito $A$ acrescenta, inclusive, que possíveis sabotagens atrapalham o processo de implantação dos frameworks. Segundo $\boldsymbol{A}$ "A cultura das pessoas [...]. Você começa, mas ficam desvirtuando".

Com relação à preparação técnica para implementar e utilizar frameworks de gestão e governança de TI os sujeitos $\boldsymbol{A}, \boldsymbol{B}, \boldsymbol{C}$ e $\boldsymbol{D}$, foram unânimes em colocar que não existe qualquer treinamento formal da equipe, e que os técnicos que tem algum treinamento o fizeram por conta própria. $O$ sujeito $\boldsymbol{B}$ demonstrou que existe um interesse em treinar a equipe de forma a melhorar a capacitação, mas que este processo ainda aguardava aprovação pela direção. $O$ sujeito $\boldsymbol{C}$ declara tacitamente que "a capacitação é o principal empecilho na implantação [de frameworks de TI]". Já o sujeito $\boldsymbol{D}$ declara que "a principal dificuldade é a falta de conhecimento técnico, e que para efetuar uma implantação seria necessária a contratação de uma consultoria externa".

Situação oposta à apresentada pelo sujeito $E$. Este colocou que é uma prática da empresa investir na capacitação dos funcionários citando, inclusive, um plano de 
carreiras. Segundo o sujeito $E$ :

Para determinados cargos, eu preciso que a pessoa tenha o mínimo desejável para ocupar o cargo [...], a partir daí é feita uma escala de produtividade onde o profissional vai galgando as posições, passa para assistente, para analista júnior... e a partir daí para coordenador. Na medida que o profissional vai passando por esses cargos, nós temos um processo de treinamento interno, e temos um processo de avaliação.

Finalmente existe uma falta de interesse dos executivos em entender como gerenciar o setor de TI. Não são utilizadas as ferramentas necessárias para que os gestores do negócio possam participar da gestão de TI, e estes não têm interesse em se qualificar de forma a suprir essa deficiência. Isto pode ser observado na colocação do sujeito $\boldsymbol{B}$, quando este declara que:

Eles têm sido bem positivos no que eu tenho levado para eles, e eu acredito que com o COBIT não vai ser diferente, eles podem não participar, mas vão apoiar a implementação.

O COBIT é um framework que prega a participação da diretoria na governança de $\mathrm{Tl}$, mas, segundo o sujeito $\mathbf{B}$ da pesquisa, "eles podem não participar". Apoiando essa análise o sujeito $\boldsymbol{A}$ coloca que:

No início a diretoria até perguntou, questionou, mas até sem ter base de fundamento do que era a ferramenta, eu acho que escutou alguém comentar, algum outro empresário comentar[...], mas eu acho que eles não dão a importância que deve ser dada.

\subsection{Determinantes da não utilização de frameworks de gestão e/ou governança de TI}

Após análises das declarações dos sujeitos participantes da pesquisa, quatro pontos foram identificados como sendo determinantes da baixa utilização de frameworks de gestão e/ou governança de $\mathrm{TI}$, (1) o distanciamento da direção da empresa com relação ao setor de $\mathrm{TI}$, (2) a cultura organizacional que dificulta a implantação de novos métodos de trabalho, (3) a baixa qualificação dos profissionais de $\mathrm{TI}$ em relação aos frameworks de gestão e/ou governança, e (4) a falta de qualificação da direção da empresa em relação aos frameworks de gestão e/ou 
governança e a falta de interesse em realizar essa capacitação.

\subsubsection{O distanciamento da direção da empresa com relação ao setor de TI}

Pauli e Barreto (2011) destacam que o distanciamento entre negócios e TI é o resultado de problemas de relacionamento entre os usuários finais e o pessoal técnico. Existem as dificuldades de comunicação, entendimento, parceria e interação entre as áreas, fazendo com que esse distanciamento venha a surgir desde o início da utilização das ferramentas de tecnologia.

Corroborando com a afirmação feita por Pauli e Barreto (2011), Mann (2002) afirma que existe um desalinhamento entre $\mathrm{TI}$ e o negócio, que leva ao distanciamento entre as partes e a situações de ineficiência na entrega de serviços.

Esse distanciamento pode ser verificado no presente estudo. Fato interessante é que a utilização dos frameworks de gestão e/ou governança de TI tem como objetivo reduzir essa lacuna, então o distanciamento dificulta a implantação e a implantação diminuiria a distância. Então a questão é, quem deveria vir primeiro?

\subsubsection{A cultura organizacional}

Salles et al. (2013) afirmam que a adoção de medidas de gestão e controle de TI pode encontrar dificuldades em situações nas quais a cultura organizacional é enrijecida. Weiss e Bernardes (2014) corroboram com essa constatação ao afirmarem que características culturais da empresa devem ser levadas em consideração na implantação de ferramentas de gestão de TI, pois a solução passa, não somente pela documentação dos processos e práticas, mas também pela implementação de uma nova forma de trabalho.

Essa dificuldade em implementar uma nova forma de trabalho, a resistência a mudanças e possíveis sabotagens nos processos de implantação, foram percebidas nas declarações dos participantes da pesquisa. A cultura "engessada" e a dificuldade em lidar com mudanças foi um dos pontos importantes da não adoção de frameworks de gestão e/ou governança de TI.

Reconhecer que as mudanças organizacionais são processos complexos, mas que podem trazer grandes benefícios pode ser um ponto facilitador na adoção 
de frameworks de gestão e/ou governança de TI nas organizações.

\subsubsection{A baixa qualificação dos profissionais de TI}

A baixa qualificação dos profissionais de TI que deveriam promover a utilização das ferramentas de gestão foi percebida no estudo. Em vários momentos foi possível identificar que a baixa qualificação é um dos motivos pelos quais não são adotados frameworks mundialmente reconhecidos como sendo de melhores práticas.

Resultados semelhantes foram encontrados por Da Silva et al. (2008). Para os autores é difícil solucionar os problemas relacionados aos serviços prestados pelo setor de $\mathrm{Tl}$, sem que os profissionais que atuam nessa área estejam devidamente capacitados. Capacitação essa que tem o direcionamento gerencial de forma que estrutura técnica possa ser utilizada pela organização em sua plenitude.

Aqui faz-se necessária a conscientização das entidades formadoras de que o lado gerencial da formação do profissional de TI deve ter seu espaço na grade curricular. Adicionalmente, o profissional de TI tem que perceber a importância da gestão no seu trabalho diário e no seu crescimento profissional.

\subsubsection{A falta qualificação da direção em relação à gestão e/ou governança de TI}

A qualificação da alta gestão nas ferramentas que possibilitarão um melhor direcionamento da $\mathrm{TI}$ ainda é um assunto pouco abordado na literatura. Em geral, a indicação da necessidade de qualificação é feita em relação à equipe de $\mathrm{Tl}$, mas nada se fala em relação ao entendimento da alta gestão em relação às tarefas realizadas pela equipe de $\mathrm{TI}$.

O setor de TI ainda é visto na organização como um "ser à parte", o qual não se entende como vive, o que faz, nem qual o resultado das suas ações. Entender os processos de $\mathrm{Tl}$, como gerenciá-los e controla-los, não significa que o gestor organizacional tenha que ser formado em TI. Vários são os cursos que apresentam os fundamentos de gestão e governança de $\mathrm{TI}$, que são direcionados ao público geral da administração. Falta, de fato, o interesse em adquirir esse conhecimento. 
Essa falta de interesse da direção nas atividades de TI pode ser percebida nas declarações dos sujeitos da pesquisa quando $\mathbf{A}$ declara que "[a diretoria] não tem base de fundamento do que seja", ou quando B afirma que "[farei uma] apresentação de como é que funciona, [...] e só depois disso é que eu vou saber o quanto é que o pessoal vai ficar disposto", ou, segundo C, que "Para a direção este assunto é indiferente". Apenas o sujeito $\mathrm{E}$, que tem ferramentas de gestão e governança de $\mathrm{TI}$ implantadas, afirma que "Existe um complexo de ferramentas interligadas que permitem o acompanhamento da direção".

\section{Conclusão}

Neste estudo avaliamos a utilização de frameworks de governança e gestão TI. A utilização de frameworks consagrados como ITIL, COBIT ou outros internacionalmente conhecidos, é indicada e verificada em estudos que mediram as eficácias destes. Mesmo assim identificamos que grande parte das empresas, pequenas médias e grandes, não implementam esses frameworks, preferindo utilizar metodologias próprias e desenvolvidas a partir de metodologias de tentativa e erro.

Foram identificados nesse trabalho quatro possíveis razões para que as empresas não adotem frameworks de gestão e/ou governança internacionalmente conhecidos, e optem por continuar a utilizar os chamados "padrões próprios". São elas:

APÊNDICE A - Distanciamento da direção da empresa com relação ao setor de TI

APÊNDICE B - Cultura organizacional, dificultando a implantação de novos métodos de trabalho;

APÊNDICE C - Baixa qualificação dos profissionais de $\mathrm{TI}$ em relação aos frameworks de gestão e/ou governança;

APÊNDICE D - Falta de capacitação dos executivos da empresa em relação aos frameworks de gestão e/ou governança e a falta de interesse em realizar essa capacitação.

A gestão e governança de TI é um aspecto que deve ser tratado de maneira séria nas empresas. Não é mais possível que Tecnologia da Informação seja relegada a segundo plano como sendo apenas responsabilidade dos "técnicos", e no caso de 
falhas no cumprimento das suas atribuições assumir toda a responsabilidade, pois " $\mathrm{TI}$ é um problema que não tem solução". A baixa aderência a padrões internacionalmente reconhecidos é um aspecto que revela a falta de preparo da direção e dos profissionais de $\mathrm{TI}$.

Gerir e governar TI é responsabilidade da alta direção que não pode se omitir dessa tarefa. Não é necessário ter conhecimento técnico para desempenhar estas funções, os frameworks de gestão e governança estão à disposição para aqueles que desejarem realizar um bom trabalho, mas é necessário que a direção assuma o seu papel, e também a equipe de $\mathrm{Tl}$, de forma que o trabalho seja em conjunto e que o resultado foque nos objetivos da organização.

Os cursos técnicos e superiores, tanto da área de TI quanto da área de administração, precisam formar profissionais que tenham a capacidade de ver o setor de TI como um ativo empresarial, e possam aproveitá-lo como um diferencial competitivo. Muitas são as situações nas quais um profissional assume um departamento de TI sem ter tido o treinamento necessário de gestão para que a operação deste seja a melhor possível. Além disso, é necessário que os profissionais busquem treinamento e especialização, de forma que uma formação continuada permita a melhoria profissional e, consequentemente, melhoria de performance e eficiência organizacional.

\subsection{Sugestões para estudos futuros}

A partir dos achados do presente trabalho, algumas indicações de futuros estudos podem ser realizadas. Primeiro, como a capacitação, tanto da equipe de TI quanto da direção da organização, poderia impactar na adoção e no retorno que os frameworks de melhores práticas em TI proporcionariam?

Adicionalmente, é possível analisar o processo de implantação das ferramentas de melhores práticas, utilizando uma abordagem na qual tanto a TI quanto a gestão da organização participassem de forma conjunta. Assim, poderia ser quebrado o ciclo no qual a utilização das ferramentas possibilita a aproximação da TI com o negócio.

Um potencial estudo a ser realizado é a aplicação de um questionário fechado, previamente validado, contendo as dimensões dos frameworks de gestão e 
governança, de forma que pudesse ser avaliado o nível de utilização desses processos, mesmo em situações de informalidade.

Finalmente, em relação à questão da cultura organizacional, quais os tipos de cultura organizacional são mais resistentes à implantação dos frameworks de gestão e governança e quais os processos poderiam ser utilizados para vencer essas resistências?

\subsection{Limitações}

Durante o processo de realização do presente trabalho, ocorreram algumas limitações. Primeiro, o acesso a um maior número de organizações que possibilitasse uma melhor visão da utilização de frameworks de gestão e governança de $\mathrm{TI}$. Segundo, o acesso aos próprios gestores para a realização das entrevistas e a disponibilidade de tempo dos mesmos.

Outra limitação presente no estudo é a utilização do questionário direcionado. Apesar de indicado ao propósito do estudo, que foi o levantamento da utilização de frameworks de gestão ou governança de $\mathrm{TI}$, o mesmo não possibilita a utilização de análises estatísticas avançadas, o que pode vir a ser realizado com um questionário fechado previamente validado.

Finalmente, foi difícil ter acesso aos gestores de TI de forma que a etapa das entrevistas pudesse ter sido realizada. Não foi possível, por exemplo, entrevistar um gestor de TI de uma empresa da área de educação, ou um gestor do setor público. Apesar da saturação obtida na etapa qualitativa, a participação destes profissionais poderia enriquecer o estudo.

\section{Referências}

Bianchi, I. S. (2015). Frameworks e Práticas de Governança de TI Para Universidades: Oportunidades de Pesquisa Baseadas em uma Revisão Sistemática da Literatura. In: 12th CONTECSI International Conference on Information Systems and Technology Management (São Paulo).

Broussard, F. W., \& Tero, V. (2007). Configuration and change management for IT compliance and risk management: The tripwire approach. White Paper. IDC. 
Cappelle, M. C. A., Melo, M. C. D. O. L., \& Gonçalves, C. A. (2011). Análise de conteúdo e análise de discurso nas ciências sociais. Organizações rurais \& agroindustriais, 5(1).

Cater-Steel, A., Tan, W. G., \& Toleman, M. (2006). Challenge of adopting multiple process improvement frameworks. In Proceedings of 14th European conference on information systems (ECIS 2006), pp. 1375-1386. European Conference on Information Systems.

Creamer, G., \& Freund, Y. (2010). Learning a board Balanced Scorecard to improve corporate performance. Decision Support Systems, 49(4), 365-385.

da Silva, L. S., Lima, R. S., \& Pires, T. C. F. R. (2008). Gestão de Service Desk baseado no modelo ITIL: proposta de implementação no Tribunal Regional do Trabalho da $8^{\underline{a}}$ Região. In Congresso Brasileiro de TI \& Telecom (INFOBRASIL), Fortaleza.

Alcantara, R. M. P. D. (2007). Raciocínio qualitativo no gerenciamento de serviços de tecnologia da informação. Mestrado. Brasília. UnB.

Gil, A. C. (2010). Amostragem na pesquisa social. Gil AC, organizador. Métodos e técnicas de pesquisa social (6a ed.). São Paulo: Atlas, pp.90-109.

Iden, J., \& Eikebrokk, T. R. (2013). Implementing IT Service Management: A systematic literature review. International Journal of Information Management, 33(3), 512-523.

ITGI (2003). Board briefing on IT governance (2a ed.). IT Governance Institute.

ITGI (2007). CobiT - Control Objectives for Information and related Technology (4aed.). Rolling Meadows: ISACA. Recuperado de http://www.isaca.org/Knowledge-Center/cobit/Documents/CobiT 4.1.pdf

ITGI (2012). CobiT 5 - Modelo Corporativo para Governança e Gestão de TI da Organização. ISACA. http://www.isaca.org/cobit/Pages/CobitFramework.aspx.

Kaur, R., \& Sengupta, J. (2013). Software process models and analysis on failure of software development projects. arXiv preprint arXiv:1306.1068.

Laurindo, F. J. B. (2008). Tecnologia da informação: planejamento e gestão de estratégias. Atlas.

Lunardi, G. L., Becker, J. L., \& Maçada, A. C. G. (2010). Governança de TI e suas Implicações para a Gestão da TI: Um Estudo Acerca da Percepção dos Executivos. XXXIV Encontro da ANPAD-EnANPAD. Rio de Janeiro, 6. 
Lunardi, G., Dolci, P., Maçada, A. C., \& Becker, J. L. (2014). Análise dos mecanismos de governança de $\mathrm{Tl}$ mais difundidos entre as empresas brasileiras. Revista Alcance, 21(1).

Lunardi, G. L., Becker, J. L., Maçada, A. C. G., \& Dolci, P. C. (2014). The impact of adopting IT governance on financial performance: An empirical analysis among Brazilian firms. International Journal of Accounting Information Systems, 15(1), 66-81.

Lyra, M. R. (2013). A contribuição da Arquitetura da Informação para o Gerenciamento de Serviços de TI. (Tese de doutorado. Universidade de Brasília, UnB, Brasília, DF, Brasil. ).

Magalhães, I. L., \& Pinheiro, W. B. (2007). Gerenciamento de serviços de TI na prática: uma abordagem com base na ITIL: inclui ISO/IEC 20.000 e IT Flex. Novatec Editora.

Manzini, E. J. (1990). A entrevista na pesquisa social. Didática, 26, 149-158.

Ngulube, P., Mathipa, E. R., \& Gumbo, M. T. (2015). Theoretical and conceptual frameworks in the social and management sciences. Addressing research challenges: making headway for developing researchers, 43-66.

OGC (2007). The Official Introduction to the ITIL Service Lifecycle (ITIL). UK: The Stationery Office.

Orr, A. T., \& Britain, G. (2011). Introduction to the ITIL Service Lifecycle. The Stationery Office.

Pauli, S.; Barreto, L.F.B.P.M (2011). Alinhamento Estratégico entre Negócios e TI: Uma Análise da Percepção de Atuais e Futuros Administradores de Empresas. EnANPAD, $35^{\circ}$. Anais... Rio de Janeiro: ANPAD.

Pereira, R. F. D. S., \& Silva, M. M. D. (2010). A maturity model for implementing ITIL v3. In Proceedings of the 2010 6th World Congress on Services (pp. 399406). IEEE Computer Society.

Salles, A. C., Alves, A. P. F., Dolci, D. B., \& Lunardi, G. L. (2013). Adoção de práticas de TI verde nas organizações: Um estudo baseado em mini casos. Anais. Bento Gonçalves, EnADI, v. 2013.

Talea, A., El Guemmat, K., Talea, M., \& Tragha, A. (2015). Towards A New System of Selecting Information Systems Frameworks. International Journal, 5(12).

Tarantino, A. (2008). Governance, risk, and compliance handbook: technology, finance, environmental, and international guidance and best practices. John Wiley \& Sons. 
THE STANDISH GROUP (2014). CHAOS Summary 2014. The Standish Group International, Boston.

Weill, P., \& Ross, J. W. (2006). Governança de Tl, tecnologia da informação. São Paulo: M. Books do Brasil.

Weill, P., \& Ross, J. (2005). A matrixed approach to designing IT governance. MIT Sloan Management Review, 46(2), 26.

Weiss, M. C., \& Bernardes, R. C. (2014). As práticas de governança e gerenciamento de serviços de TI como vetor para a melhoria do desempenho empresarial: Estudo de caso em uma empresa atacadista. Gestão \& Planejamento-G\&P, 15(1).

Zaitar, Y., \& Ouzarf, M. (2012). ERP Projects: Key success factors and risk of failure a proposed model of governance of enterprise resource planning. International Journal of Computer Applications, 46(8), 34-39. 